\title{
Caracterización del sistema de seguridad de semillas en el municipio de Cruces
}

\author{
Characterization of the system of safety of seeds in the Cruces's municipality \\ Dorcas Li Puello ${ }^{*}$, Yoandris Socarras Armenteros ${ }^{1}$, Alberto López. Méndez ${ }^{1}$
}

\section{RESUMEN}

El mundo necesita constantemente aumentar la productividad de los cultivos y crear nuevas variedades mejor adaptadas para enfrentar factores ambientales y biológicos o satisfacer las necesidades de las comunidades locales. Este trabajo persigue mejorar la labor que se viene realizando en este sentido en Cuba, proponiendo una estrategia que permita aumentar la producción de los recursos fitogenéticos necesarios para garantizar una mejor seguridad alimentaria en el municipio. Para la realización del diagnóstico se tuvo en cuenta la guía metodológica para la seguridad de semillas, elaborado por la Unitarian Service Committee of Canada (USC), y se seleccionó al azar una muestra de 38 fincas distribuidas en los distintos consejos populares del municipio. Los principales resultados indican que existe diversidad de semillas en el sistema local, mientras que el sistema formal no se garantiza la diversidad, disponibilidad y calidad requeridas; se aplican diversos métodos de almacenaje para la conservación de semillas y la mayoría de los productores no producen la semilla de forma separada, con las atenciones requeridas para este tipo de producción. Se conoció que es baja la participación de mujeres y jóvenes en las actividades agrícolas y en la toma de decisiones relacionadas con la semilla. Quedó elaborado un sistema de intervenciones que redundará en el mejoramiento de la producción de semillas en el municipio.

Palabras clave: diversidad, disponibilidad, intervención, calidad, fitogenético.

\section{ABSTRACT}

The world constantly needs to increase the crop production and to create new and better adapted varieties to face environmental and biological factors or to satisfy the demands of the local communities. The main purpose of this paper is to improve the work that is being carried out in this respect in Cuba, proposing a strategy that allows an increase in the production of the phytogenetic resources so as to guarantee a better food safety in the municipality. The diagnosis was carried out taking into account the methodological guidelines for the safety of seeds elaborated by the USC of Canada and using a random sample of 38 private farms from different district councils of the municipality. The main results show that there is diversity of seeds in the local private system; however, the formal system doesn't guarantee diversity, availability and quality. It also revealed that diverse storage methods are applied for the conservation of seeds, and that most of the farmers do not produce the seed in an isolated safe way with the required culture for this type of production. On the other hand, the diagnosis also revealed that there is low participation of women and young people in agricultural activities and in the taking of decisions related with the production of seeds. As a result, a system of interventions for the improvement of safer seed productions for the local agriculture was elaborated.

Key words: diversity, availability, intervention, quality, phytogenetic.

\section{Introducción}

El concepto de seguridad alimentaria ha sido utilizado con diferentes sentidos a lo largo del tiempo. A partir de la Conferencia de Alimentación, celebrada en Roma en el año 1974, han surgido diversas definiciones lo que no le resta importancia al tema sino, por el contrario, los múltiples usos del término reflejan la naturaleza del problema alimentario.

En las últimas décadas, a pesar de los esfuerzos realizados, todavía sigue inconclusa la tarea de garantizar la seguridad alimentaria a los más pobres de forma sostenible. No se puede afirmar

\footnotetext{
1 Universidad de Cienfuegos Carlos Rafael Rodríguez, Cuba.

* Autor por correspondencia: dlipuello@ucf.edu.cu
} 
que hay seguridad alimentaria si no hay seguridad de semillas, ya que, la diversidad de semillas, diversidad en los tipos/variedades de semillas también determina la diversidad de los cultivos, lo que es clave para la construcción de un sistema de producción de alimentos frente a los impactos del cambio climático. Por tanto, es importante comprender los sistemas agrícolas de un área para evaluar cómo los cambios en los componentes y prácticas agrícolas pueden impactar a los sistemas de semillas operando en esa área.

Cuba depende de los recursos fitogenéticos para la alimentación y la agricultura de otros países para llevar a cabo sus programas de mejora fitogenética (Fernández, 2013). Su labor en el combate contra el hambre y la seguridad alimentaria de su población en medio de difíciles condiciones económicas fue reconocida por la representación de la Organización de Naciones Unidas para la Alimentación y la Agricultura (FAO), en la isla (Jiménez, 2015).

En el municipio de Cruces, se realizan grandes esfuerzos para garantizar la alimentación de sus pobladores, incentivando la ejecución de proyectos relacionados con producciones sostenibles, cadena de valores y enfrentamiento al cambio climático en su propósito de sustituir importaciones de alimentos. El área económica fundamental fue por muchos años la industria azucarera de un $83,2 \%$ de la superficie total agrícola, el $63,3 \%$ se destinaba a la caña de azúcar. A partir del proceso de reconversión de la industria azucarera la situación varía significativamente pues el municipio pasa a ser eminentemente agrícola, situación que debe enfrentar sin una tradición de trabajo en este sector.

Por tal motivo, es que se hace necesaria la realización del diagnóstico sobre la seguridad de semillas en el municipio, con vistas a trazar estrategias para contribuir al mejoramiento de la producción de alimentos en el territorio.

\section{Metodología}

El trabajo se realizó en el municipio de Cruces, provincia Cienfuegos en el período comprendido de diciembre de 2015 a febrero de 2016. Para realizar las encuestas se seleccionaron 38 productores al azar pertenecientes a los seis Consejos Populares del municipio y a diversas Cooperativas de Créditos y Servicios (CCS) y Cooperativas de Producción Agropecuaria (CPA).
Se emplearon cuatro fuentes de información y datos para los variados elementos de los análisis de los sistemas agrícolas, sistemas de semillas y seguridad de semillas:

1. Oficina Nacional de Estadísticas de Cuba (ONEI), informe de la línea base del PIAL y otros informes.

2. Discusiones de grupos focales (DGF).

3. Entrevistas a informantes clave (EIC).

4. Encuestas domiciliarias.

Se seleccionaron como informantes claves a directivos de la Asociación Nacional de Agricultores Pequeños (ANAP), Ministerios de la Agricultura (MINAG) y la Granja Agropecuaria, para la conformación de los grupos focales productores con conocimientos del sistema productivo y de los sistemas de semillas.

Durante la realización del diagnóstico se tuvo en cuenta la guía metodológica para la seguridad de semillas elaborado por la USC de Canadá (Shrestha, 2007) y validado en el 2014 en Cuba en colaboración con productores y colaboradores nacionales, regionales y municipales de Cuba.

\section{Resultados y Discusión}

\section{Análisis de los sistemas agrícolas}

El municipio de Cruces tiene una extensión de $193,35 \mathrm{~km}^{2}$. Está ubicado al noroeste de la provincia de Cienfuegos, en los $22^{\circ} 18^{\prime}$ latitud norte y $80^{\circ} 12^{\prime}$ longitud oeste. Limita al norte con Ranchuelo, al sur con Palmira, al este con Cumanayagua y por el oeste con Santa Isabel de las Lajas.

El relieve, que se caracteriza por tener presencia de llanuras onduladas o colinosas, llanuras poco desmembradas, terrazas fluviales y pequeñas colinas dispersas, siendo el punto culminante La Rioja con 137,6 $\mathrm{m}^{2}$. Los suelos que más predominan son húmicos, calcimórficos y ferralíticos.

La topografía del terreno dedicado a las actividades agrícolas en el área del proyecto abarca dos tipos de relieve: llano, y montaña, estando la mayor proporción de domicilios y de área agrícola en el llano donde la diversidad de cultivos es considerada baja y la fertilidad del suelo como mediana.

El clima en el municipio se clasifica como tropical cálido la humedad relativa media es del 
$78 \%$, la temperatura media anual es de $25.6{ }^{\circ} \mathrm{C}$. La temperatura media en los últimos cinco años es de $23,3^{\circ}$, y el promedio de precipitación ha oscilado alrededor de 112,28 mm (ONEI, 2015).

En los últimos cinco años no han ocurrido desastres naturales ni incendios forestales que pudieran afectar las cosechas, solamente se han registrado como máximo cinco meses sin lluvia lo que ha afectado a lugares que carecen de agua, en pequeñas proporciones demorando principalmente la siembra del arroz, que se realiza en diques (ONEI, 2015).

La población residente es 30.825 con una densidad poblacional de $159,4 \mathrm{hab} / \mathrm{km}^{2}$. El mayor porciento poblacional se encuentra en el centro urbano, la población rural de los Consejos Populares incluidos en el diagnóstico se caracteriza por predominar el sexo masculino, el tamaño promedio familiar es de tres personas y son pocos los domicilios liderados por mujeres (Tabla 1), (ONEI, 2015).

En el municipio de Cruces, el desempeño del suministro de las fuentes de irrigación empleadas por los productores se cataloga como regular, destacándose en mayor proporción (1.368,6 ha) la irrigación subterránea y con valores desfavorables el resto de las fuentes, debido a que Cruces carece de corrientes caudalosas de agua, lo que se atenúa con la construcción de pozos de brocal y artesianos, siendo su construcción muy factible.

Existe acceso a caminos para todas las comunidades agrícolas, en buenas condiciones y abiertos en todas las temporadas, la transportación se realiza con medios propios (caballos, volantas, carretas, tractores, camiones) y transporte local (camiones, omnibus), por lo que se considera que no existen dificultades en el transporte estatal y privado para la entrega de insumos y productos agrícolas.
En el municipio existen distintos tipos de mercados para vender y comprar productos agropecuarios como: los puntos de ventas, placitas, carretillas y un área de venta habilitada los sábados denominada feria agropecuaria donde acuden productores de diferentes formas productivas del municipio. Tanto los productores como los consumidores tienen accesos a los productos en los diferentes consejos populares.

Las principales empresas que brindan servicio a los productores en el municipio son Labiofam, Cimex y Gelma que tienen puntos de ventas de insumos agrícolas, lo que facilita un mejor acceso a los productores para garantizar las producciones y un mejor rendimiento de los cultivos, además se le asignan algunos recursos al productor que tienen contratos para la entrega de producciones priorizadas como el tabaco, su desempeño fue valorado como regular. La empresa agropecuaria Mal Tiempo, es la única encargada en el municipio de distribuir los insumos a los diferentes sistemas de producción cooperativa del municipio.

El principal sistema de tenencia de la tierra en es el usufructo, otorgado por los Decreto Ley 259 (MINJUS, 2008), y 300 (MINJUS, 2012). El $100 \%$ de las fincas encuestadas poseen cultivos temporales, mientras que solo el $31 \%$ tiene cultivos permanentes. El sexo masculino tiene mayor representatividad en cuanto al uso y el sistema tenencia de la tierra en el municipio, igualmente sucede con la tenencia de ganado, la mujer solo participa en la crianza de cerdos y aves de corral.

Existe diversidad en la crianza de ganado doméstico, predominan las aves en los tres municipios, los cerdos y el ganado bovino. La diversidad de razas de animales es muy baja, pues la mayoría de los productores cuentan con una sola raza de las diferentes especies de ganado.

Tabla 1. Composición de la población por sexo, tamaño promedio familiar y proporción de domicilios liderados por mujeres.

\begin{tabular}{|c|c|c|c|c|c|}
\hline \multirow{2}{*}{ Consejos populares } & \multicolumn{3}{|c|}{ Población por sexo } & \multirow{2}{*}{$\begin{array}{c}\text { Tamaño promedio } \\
\text { familiar }\end{array}$} & \multirow{2}{*}{$\begin{array}{c}\% \text { de domicilios } \\
\text { liderados por mujeres }\end{array}$} \\
\hline & Hombres & Mujeres & Ambos & & \\
\hline Peñasco & 7 & 3 & 10 & 2 & \\
\hline San José & 11 & 4 & 15 & 2 & 1 \\
\hline Mal Tiempo & 8 & 6 & 14 & 3 & \\
\hline El No Chicharrones & 28 & 14 & 42 & 4 & 1 \\
\hline Marta Abreus & 7 & 5 & 12 & 4 & \\
\hline Potrerillo & 20 & 13 & 33 & 4 & \\
\hline
\end{tabular}

Fuente: Encuesta domiciliaria. 
La disponibilidad de mano de obra familiar es alta, ya que en su mayoría todos los miembros de la familia participan en las actividades agrícolas, no sucede así con la mano de obra contratada que en ocasiones se dificulta por la lejanía del lugar de la cosecha. La estrategia que se utiliza para acceder a ella, es aumentar la tarifa de pago que, generalmente oscila alrededor de los 50 CUP por día, y ofrecer facilidades para la transportación de los contratados; de las labores que se realizan a los cultivos la que más se ve afectada es el desyerbe.

En el municipio, la principal fuente de ingreso para los productores es la venta de granos, seguido por las verduras, siendo más baja la proporción de domicilios que se dedican a la producción de derivados de origen animal (Gráfico 1).

Los préstamos bancarios se realizan mediante Banco Nacional de Crédito y Comercio (BANDEC), a la que tienen acceso todos los productores, principalmente para la obtención insumos como semillas, plaguicidas, fertilizantes químicos y herbicidas, aunque solo el $13 \%$ de los domicilios encuestados han solicitan préstamos bancarios para sostener sus producciones.

Con respecto al arreglo de modos de producción (unidades de producción) la mayoría de los domicilios encuestados se encuentran asociados a CCS, ocupando la menor superficie la granja estatal. No existe en el municipio ninguna estación de investigación, pero si se realizan actividades de investigación vinculadas con trabajos de cursos y de diploma de los estudiantes de la Universidad de Cienfuegos (UCF), teniendo el Centro Universitario Municipal (CUM) como facilitador de cada una de ellas y el Centro politécnico José Cruz Gastón.

Los productores participan en las actividades de investigación y, a su vez, se capacitan en los talleres que se realizan en el municipio, el diagnóstico de sus necesidades de capacitación se realizan en estos mismos talleres y en visitas efectuadas a los productores y en el marco de las reuniones de balance de sus respectivas cooperativas. Este marco es aprovechado para diseminar nuevas tecnologías en relación con la producción y conservación de semillas mediante el Proyecto de Innovación Agrícola Local (PIAL) que ha venido realizando una gran labor durante varios años.

En el municipio tienen lugar múltiples actividades de extensión agrícola, que involucran a hombres, mujeres, niñas y niños, entre las que se encuentran las organizadas por el sistema de la agricultura, el CUM, el Ministerio de Ciencia,

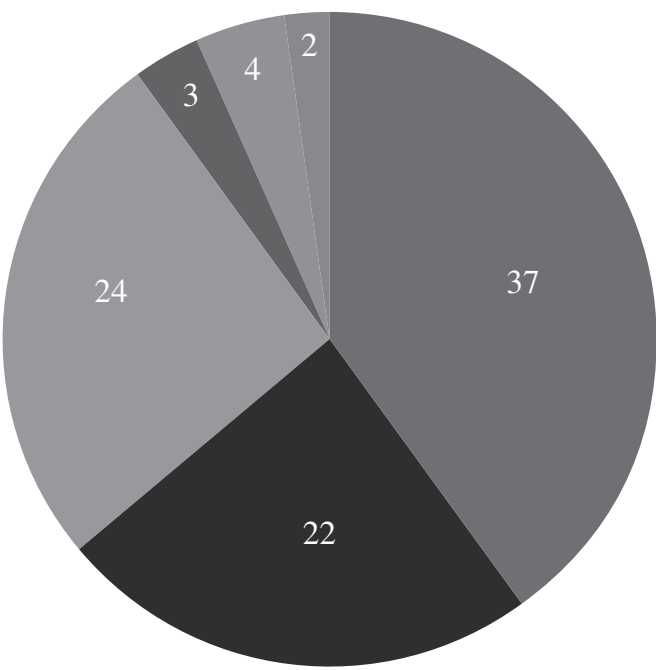

Venta de granos

Venta de frutas $\square$ Venta de verduras

Venta de semillas Otras fuentes agrícolas

Animales y productos derivados

Fuente: Encuesta domiciliaria.

Gráfico 1. Principales fuentes de ingreso en el municipio. 
Tecnología y Medio Ambiente (CITMA), Educación, la Universidad y organizaciones no gubernamentales (ONGs) relacionadas con las actividades agrícolas como Asociación Cubana de Técnicos Agrícolas y Forestales (ACTAF), Asociación Nacional de Agricultores Pequeños (ANAP) y Asociación Cubana de Producción Animal (ACPA). También las capacitaciones y actividades de aprendizaje y extensión (semillas, tecnologías) ejecutadas por los proyectos que impactan en el municipio como PIAL, Hábitat y PRODEL.

Los agricultores en el municipio se organizan en la ANAP, en el territorio existen hasta el momento siete Grupos de Innovación Agrícola Local (GIALs) distribuidos en los diferentes consejos populares del municipio operando en este sistema agrícola a los que se vinculan 25 domicilios. Todos los encuestados pertenecen a la ANAP.

Las ONGs que prestan servicios en el municipio son la ACTAF, ACPA, ANAP y proyectos internacionales como el PIAL, que en conjunto con el CUM capacita a los productores en cuanto a las buenas prácticas a introducir en las fincas, mediante el desarrollo de talleres, eventos, y la entrega de insumos a varios productores que facilitan el manejo y la conservación de semillas.

Según información brindada por productores y decisores, dentro de los principales problemas detectados en cuanto al sistema agrícola existente se encuentran en orden de prioridad:

- Deficiente comercialización de producciones agrícolas.

- Falta de insumos (semillas, fertilizantes, biofertilizantes, insecticidas, fungicidas, herbicidas, combustible y sistemas de riego).

- Degradación de los suelos.

- Demora en la entrega de paquetes tecnológicos a productores.

- No existen mecanismos de certificación de semillas.

\section{Análisis de sistemas de semillas}

El sistema de la agricultura tradicional campesina cubana, especialmente en lo referente a la producción de semillas, no existe aislado del sistema convencional (Castiñeiras et al., 2012). En el municipio predomina los alimentos básicos (cultivos: maíz, frijol, arroz, yuca y plátano), con mayor extensión de tierra, no sucediendo lo mismo con los cultivos comerciales, pues son pocos los productores que se dedican a ello. Según los resultados de las entrevistas se debe a que no existe cultura sobre los cultivos comerciales y los productores tienen pequeñas extensiones de tierra por lo que prefieren utilizarlos en la producción alimentos básicos.

Las encuestas realizadas a los productores, demostraron que el frijol, arroz, maíz, son los cultivares que mayor número y tipos de variedades presentan. También presentan la mayor proporción de domicilios y de superficie a sembrar. Durante el diagnóstico de la diversidad varietal de los cultivos clave se conoció que de manera general los agricultores no están familiarizados con los nombres de las variedades de las especies que siembran, predominando los nombres locales, que difieren en el mismo municipio, lo que no permite hacer un registro de la diversidad varietal.

Los productores plantean que existen pocas variedades, porque a pesar que se ha distribuido varias variedades de semillas de granos como producto de las acciones del proyecto PIAL, los productores han seleccionado la que mejor se ha adaptado a las características del suelo y la de mayor rendimiento, lo que ha conllevado a que la diversidad varietal en los últimos años no ha variado.

Al realizar el análisis de la diversidad de cultivos, se conoció que son muchos los domicilios con áreas pequeñas dedicados a la alimentación básica; sin embargo, existen grandes áreas con pocos domicilios dedicados a cultivos comerciales (Tabla 2).

Tabla 2. Tipos/categorías de productos cultivados por los agricultores en el municipio.

\begin{tabular}{llcc}
\hline Categorías de cultivos & \multicolumn{1}{c}{ Cultivos } & Proporción de domicilios & Proporción de superficie (ha) \\
\hline Alimentos básicos & Maíz, arroz, frijol, yuca, plátano malanga, boniato & 39 & 279,26 \\
Hortalizas & Tomate, ají, pepino, col, lechuga, calabaza & 22 & 13,99 \\
Condimentos & Ajo, cebolla & 12 & 3,25 \\
Frutas & Mango, fruta bomba, guayaba, naranja, aguacate & 24 & 40,48 \\
Cultivos comerciales & Caña, tabaco & 6 & 1,7 \\
\hline
\end{tabular}

Fuente: Encuesta domiciliaria. 
Los productores plantean en cuanto a los cultivos como la malanga (Colocasia esculenta; (L) Schott) y el Boniato (Ipomoea batatas (L) Lam), que se ha perdido la tradición, debido, en el caso específico del cultivo de la malanga presenta un ciclo fenológico largo, y el boniato por ser muy vulnerable a las plagas y enfermedades.

El sistema no formal o local de producción de semillas juega un papel fundamental en los cultivos del plátano (Musa paradisiaca), yuca (Manihot esculenta. Crantz) y Boniato (Ipomoea batatas (L) Lam) de los que se producen el $100 \%$ por esta vía. En otros sectores, como el caso de las hortalizas se producen a menor escala semillas de tomate (Solanum lycopersicum L.), pimiento (Capsicum annuиm L.,), pepino (Cucumis sativus L.,) y Calabaza (Cucubita L.). Los granos son producidos por un grupo importante de ellos para el autoconsumo familiar por el sistema no formal. La falta de calidad y diversidad varietal de las semillas ha impactado negativamente en la productividad de estos cultivos. Al mismo tiempo, no hay garantía fitosanitaria ni incentivos productivos para aquellos campesinos que se dedican a mejorar la calidad de las semillas de sus variedades.

En el sistema local de semillas los productores producen las semillas en conjunto con los consumidos como grano, y casi no utilizan intermediarios para comercializar las semillas, se realiza directamente con el productor que la solicite.

En la provincia de Cienfuegos, existe una empresa provincial de semillas, encargada de la venta de semillas a los productores, pero existen algunas deficiencias en su funcionamiento como es la lentitud del proceso y la calidad requerida, además que no siempre existe diversidad de variedades y algunos precios son elevados y el productor no puede acceder a ella. El productor de tabaco recibe la semilla por el sistema formal, ya que esta producción está contratada con vistas a la sustitución de importaciones y exportaciones. El sistema formal de semillas en el municipio, según el Ministerio de la Agricultura (MINAG), no abarca todos los cultivos que se siembran en el territorio, cuenta con 23,2 ha, para la producción de semillas, y solo cinco productores se dedican a esta labor, los cultivos que están incluidos en el programa de producción de semilla son: el arroz (Oryza sativa), boniato, yuca y frijol (Phaseolus vulgaris).
La semilla producida por el sistema formal de semilla se utiliza para el autoconsumo del productor en un $20 \%$ y el resto para su comercialización directamente a la empresa de semillas, sin necesidad que exista intermediario.

El proceso para que los productores de las Cooperativa de Créditos y Servicios (CCS), Cooperativa de Producción Agropecuaria (CPA) $\mathrm{y}$ otras fincas individuales accedan a las semillas es mediante contrato empresa-unidad productiva.

La distribución de las semillas a los productores se gestiona por medio de la empresa conjuntamente con la dirección de las unidades productivas, pero la oferta de semillas aún es insuficiente para satisfacer la demanda. En el territorio cada productor, por tradición, tiene conocimientos empíricos en cuanto a la producción y conservación de semillas, con excepción de los cultivos comerciales que se adquieren en el mercado.

En el análisis específico de los cultivos claves, se puede apreciar que predomina como fuente de obtención de las semillas, donde el productor lo obtiene, principalmente, en los granos, no ocurriendo lo mismo con la col (Brassica oleracea var. Capitata), la cebolla (Allium cepa) y el tabaco (Nicotiana tabacum) que son adquiridos en el mercado y el café (Coffea arabica) que se adquiere en otra comunidad.

Para seleccionar las semillas, los productores utilizan con mayor frecuencia varios criterios con los que hasta el momento han obtenido buenos resultados como son: plantas más vigorosas, libre de plagas y selección del fruto más sano y de mayor tamaño, entre otros.

El tratamiento de semillas se realiza mediante métodos tradicionales como el secado al sol y en el menor de los casos, se utiliza tratamientos químicos, estos son utilizados para garantizar que el almacenaje sea eficiente.

En el almacenamiento de las semillas todos los productores utilizan en el caso de los granos, tanques herméticos; para la semilla de tomate y otras hortalizas utilizan pomos herméticos y los tubérculos son almacenados a la sombra, solo el $45 \%$ de los productores utilizan, además, productos químicos para prevenir que no sean atacados por plagas y enfermedades.

En la selección de las semillas los productores utilizan prácticas tanto antes de la cosecha como después, pocos son los que utilizan parcelas separadas, predomina la selección de plantas y frutos. 
Existen en el país leyes y reglamentos relacionadas con la producción y distribución de las semillas, que regulan el proceso del registro de estas, ello permite que semillas registradas se puedan guardar y resembrar por parte de los productores, también posibilitan la venta a nivel local entre productores.

Se pudo comprobar que el hombre es el que tiene el papel predominante en cada una de las actividades que se realizan en cuanto al manejo, ya sea en la toma de decisiones o en las actividades relacionadas con las semillas, el grado de participación de la mujer se centra en su mayoría en el tratamiento de las semillas y el almacenaje, y en el caso de los jóvenes predomina la ayuda en la selección y tratamientos en el menor de las casos.

Los resultados muestran que aún falta lograr un mayor grado de participación de las mujeres, jóvenes y niños en la toma de decisiones en las actividades relacionadas con la semilla.

Se detecta como problemas asociados con los sistemas de semillas locales en orden de prioridad:

- Utilización del grano como semilla.

- No existe un banco municipal de semillas.

- Poco acceso a variedades de semillas de calidad.

- Pocas condiciones óptimas para el almacenamiento se las semillas.

En cuanto al sistema formal de semillas se enumeran como problemas:

- Baja calidad de las semillas.

- Altos precios.

- Baja diversidad.

- Baja pureza varietal.

\section{Análisis de la seguridad de las semillas}

En la discusión de los grupos focales se analizó la seguridad de semillas en cuanto a disponibilidad, acceso y calidad, de manera general se pudo comprobar que las frutas y las hortalizas son los cultivos que tienen seguridad de semilla, mientras que el resto de los cultivos no tienen seguridad.

En el análisis realizado por los grupos focales se pudo constatar la ausencia de especies con estado B de seguridad de semillas (disponibilidad y calidad); sin embargo, el análisis evidencia que existe seguridad de semillas en el sistema local en la mayor parte de los cultivos claves (arroz, frijol, maíz y plátano).

En el área diagnosticada, no hay disponibilidad, ni acceso, ni calidad en semillas de boniato (Ipomoea batatas (L) Lam) y malanga (Colocasia esculenta; (L) Schott), es decir, que no hay seguridad de semillas para estos cultivos por lo que entre los desafíos para alcanzarlas se encuentra realizar acciones que conduzcan a la obtención y/o producción de estas semillas con la calidad requerida y establecer mecanismos para lograr tener disponibilidad, acceso y calidad a ellas.

El análisis de la seguridad de semillas empleando una matriz de cuatro celdas o cuadrantes permite establecer las relaciones entre la disponibilidad y acceso a la semilla con base en la ubicación de los cultivos y/o variedades en cada cuadrante, lo que ofrece una visión estratégica de la situación real del entorno del diagnóstico con respecto a la seguridad de semilla de diferentes cultivos y variedades.

El análisis realizado demostró que hay alta disponibilidad de semillas de plátano y ajo para satisfacer la alta demanda, lo que constituye una fortaleza, mientras la disponibilidad de semillas de malanga, y boniato siendo también baja su demanda siendo esta situación una debilidad del sistema que se debe superar.

El análisis de la situación de las variedades demuestra que se requiere incrementar la diversidad varietal, y trazar una estrategia para incentivar la producción de malanga y boniato.

Las fuentes de semillas disponibles para los agricultores por categorías de cultivos proceden, de la que el mismo productor produce. No hay establecido en el área del diagnóstico bancos de semillas para ninguna de las categorías de cultivos, dando como resultado que las encuestas no reflejen la obtención de semillas por este medio.

La disponibilidad de semillas para la mayoría de los cultivos en el territorio proviene de la que el propio productor conserva, seguido de la que obtiene en la misma comunidad, en el municipio no se produce semilla de cebolla, tabaco y col, por lo que son adquiridos por los productores en el mercado (Tabla 3). En años "malos" se activan, mayormente las reservas de semillas propias con un pequeño aporte de las producidas por otros agricultores de la misma comunidad.

El modo de acceso a las semillas para todas las categorías de cultivos (alimentos básicos, hortalizas, 
Tabla 3. Fuentes de semillas producidas y usadas para la producción de semillas por diferentes categorías de cultivos.

\begin{tabular}{|c|c|c|c|c|c|}
\hline \multirow[b]{2}{*}{ Categorías de cultivos } & \multicolumn{5}{|c|}{ Proporción de semillas de varias fuentes } \\
\hline & Semillas propias & $\begin{array}{l}\text { Agricultores de la } \\
\text { misma comunidad }\end{array}$ & $\begin{array}{l}\text { Agricultores de } \\
\text { otra comunidad }\end{array}$ & Mercado & $\begin{array}{l}\text { Apoyo de } \\
\text { OG/ONG }\end{array}$ \\
\hline Alimentos básicos & 83 & 5 & & 7 & 5 \\
\hline Hortalizas & 93 & & & 7 & \\
\hline Condimentos & 85 & & 5 & 10 & \\
\hline Frutas & 80 & 5 & 5 & 10 & \\
\hline Cultivos comerciales & 5 & 5 & 5 & 85 & \\
\hline
\end{tabular}

Fuente: Encuesta domiciliaria.

condimentos, frutas y cultivos comerciales) es en mayor proporción mediante el intercambio/ trueque y en menos proporción en como regalo entre los agricultores. La compra de semillas es de importancia en los cultivos comerciales y condimentos.

El acceso a fuentes externas de semillas para los cultivos clave es fundamentalmente por la vía del intercambio/trueque, seguido por la compra. La compra de semillas es de importancia en la col, cebolla, tabaco y ajo. El apoyo de OG/ONGs se evidencia en solo en el frijol tomate.

La venta de semillas por parte del Estado es considerada como regular, mientras que la disponibilidad de semilla en la comunidad es buena independientemente de la forma en que se adquiere la semilla.

Los encuestados consideran que la calidad de la semilla propia de los cultivos clave como alta, al igual que la que se obtiene en la comunidad, no siendo así el criterio relativo a la semilla del tomate que se obtiene en el mercado.

Los encuestados al referirse a la pureza física de sus semillas la clasifican como muy alta, excepto el caso del maíz, debido a que ese cultivo fácilmente se cruza si existe otra variedad que se siembra cerca, ellos refieren que sus cultivos no están libres de plagas y enfermedades, aunque toman las medidas para minimizar sus efectos.

En la buena adaptabilidad de los cultivos clave puede influye la existencia de variedades locales en manos de los agricultores y a que ellos han seleccionado la variedad en que mejor resultado obtienen. Las enfermedades de manera general afecta a todos los cultivos en el municipio. La calidad de las semillas propias y de fuentes externas se califica como buena por los encuestados.
El grado de disponibilidad de las semillas preferidas en el territorio se considera como muy alta, independientemente de la fuente de procedencia, sea semilla propia, o de la comunidad.

Entre los principales problemas que se identificaron asociados a la seguridad de semillas en el territorio se encuentra:

- La incidencia de plagas que afecta la obtención de la semilla propia ya adaptada al territorio.

- Poca disponibilidad de semillas en el sistema formal.

- Baja diversidad de variedades y especies en el sistema local de semillas.

- Pocas condiciones de almacenamiento para almacenar la semilla.

\section{Identificación de intervenciones para la seguridad de semillas}

Se diseñó un plan de intervenciones de prioridades de corto y largo plazo para mejorar la seguridad de semillas de los agricultores del municipio donde aparecen las siguientes acciones:

- Capacitación en producción de semillas.

- Especialización de áreas y fincas en la producción de semillas.

- Fitomejoramiento participativo.

- Crear comité locales de certificación de semillas.

- Incidencia en políticas públicas.

- Gestionar recursos.

- Divulgación mediante talleres, medios de difusión, visitas a productores experimentales, asesoría técnica.

- Establecer relaciones contractuales para un suministro de semillas de forma sistemática. 


\section{Conclusiones}

El análisis de los sistemas de semillas y su seguridad en el área del proyecto, brindó los siguientes resultados:

1. La diversidad en las diferentes categorías de cultivos en los últimos 10 años no han sufrido cambios significativos.

2. En la muestra encuestada existe diversidad de semillas en el sistema local y se aplican diversos métodos de almacenaje para su conservación.
3. La mayoría de los productores no producen la semilla de forma separada, con las atenciones requeridas para este tipo de producción.

4. El sistema formal de semillas no garantiza la diversidad, disponibilidad y calidad requeridas.

5. La producción de alimentos básicos está sustentada en el uso de semillas del sistema local.

6. Los agricultores se autoabastecen con sus propias semillas y además con semillas de otros agricultores de la misma comunidad.

7. La calidad de las semillas de diferentes fuentes de acceso se considera buena (germinación, pureza física y adaptación de las semillas), pero la resistencia a plagas es regular.

\section{Literatura Citada}

Castiñeiras, L.; Lianne, F.N.L.; Shagarodsky, T., \& Barrios, O. 2012. Limitaciones en el Sector Informal de semillas y su interacción con los Sistemas Formales. Agricultura Orgánica, 18 (2): 26-27.

Fernández, M.

2013. El tratado Internacional sobre recursos Fitogenéticos para la Alimentación y la Agricultura en Cuba. Recursos Naturales y Ambiente, 53: 105-109.

Jiménez, $\mathrm{S}$.

2015. Seguridad alimentaria y nutricional. Revista Cubana Salud Pública, 31 (3): 173.

Ministerio de Justicia (MINJUS)

2008. Decreto-Ley $N^{\circ}$ 259. Sobre la entrega de tierras ociosas en usufructo. Disponible en http://extwprlegs1.fao. org/docs/pdf/cub82664.pdf. Consultado: 16 de noviembre de 2015.

Ministerio de Justicia (MINJUS)

2012. Decreto Ley $N^{\circ} 300$. Sobre la entrega de tierras estatales ociosas en usufructo. Disponible en http://www.fgr.cu/sites/ default/files/Decreto\%20Ley\%20300\%20Tenencia\%20de\%20 la\%20Tierra.pdf. Consultado: 16 de noviembre de 2015.

Oficina Nacional de Estadísticas (ONEI)

2015. Anuario estadístico provincial. Disponible en http:// www.one.cu/publicaciones/provincias_masinf/cienfuegos. htm. Consultado: 3 /feb/2016.

Sharestha, P.S.

2007. Manual para el Diagnóstico se Seguridad de Semilla. USC. Ottawa, Canadá, 60 p. 
